\title{
Multipoint Variable Cycle Engine Design Using Gradient-based Optimization
}

\author{
John P. Jasa, \\ University of Michigan, Ann Arbor, MI, USA \\ Justin S. Gray, ${ }^{\dagger}$ Jonathan A. Seidel, ${ }^{\ddagger}$ \\ NASA Glenn Research Center, Cleveland, OH, USA \\ Charles A. Mader, ${ }^{\S}$ Joaquim R. R. A. Martins II \\ University of Michigan, Ann Arbor, MI, USA
}

\begin{abstract}
Supersonic aircraft are challenging to optimally design due to the widely varying constraints and flight conditions they experience. Additionally, a large number of disciplinary subsystems must be considered due to highly complex design requirements. One subsystem that has a major effect on overall performance is the engine. In this work, we construct a supersonic mixed-flow variable cycle engine and perform multipoint gradient-based optimization using this model. We see that the operational variables allow the optimizer to tailor performance at each individual flight condition, leading to better overall performance. To simulate airframe integration constraints, we run successive optimizations with increasingly restrictive inlet areas and see decreases in engine performance. This work is part of a larger effort to incorporate engine design into aero-thermal-mission optimization of a supersonic aircraft.
\end{abstract}

Nomenclature

$\begin{array}{ll}\alpha & =\text { angle of attack } \\ \gamma & =\text { flight path angle } \\ D & =\text { aircraft drag } \\ F_{n} e t & =\text { net force produced by the engine } \\ g & =\text { acceleration due to gravity } \\ L & =\text { aircraft lift } \\ m & =\text { aircraft mass } \\ r & =\text { aircraft range } \\ T & =\text { aircraft thrust } \\ T_{4} & =\text { temperature of the flow exiting the burner } \\ v & =\text { aircraft velocity } \\ \text { AOD } & =\text { after ordinance drop } \\ \text { BPR } & =\text { bypass ratio } \\ \text { CFD } & =\text { computational fluid dynamics } \\ \text { ER } & =\text { extraction ratio }\end{array}$

$\begin{array}{ll}\text { FAR } & =\text { fuel-to-air ratio } \\ \text { FPR } & =\text { fan pressure ratio } \\ \text { HPC } & =\text { high-pressure compressor } \\ \text { IGV } & =\text { inlet guide vanes } \\ \text { MDO } & =\text { multidisciplinary design optimization } \\ \text { MTOW } & =\text { maximum takeoff weight } \\ \text { NPSS } & =\text { Numerical Propulsion System Simulation } \\ \text { OPR } & =\text { overall pressure ratio } \\ \text { RTO } & =\text { rolling takeoff } \\ \text { SC } & =\text { subsonic cruise } \\ \text { SLS } & =\text { sea level static } \\ \text { TOC } & =\text { top-of-climb } \\ \text { TSFC } & =\text { thrust-specific fuel consumption } \\ \text { VABI } & =\text { variable area bypass injector } \\ \text { VCE } & =\text { variable cycle engine }\end{array}$

*Ph.D. Candidate, Department of Aerospace Engineering, AIAA Student Member ${ }^{\dagger}$ Aerospace Engineer PSA Branch, 21000 Brookpark Rd., MS 5-11; AIAA Member ¥Aerospace Engineer PSA Branch, 21000 Brookpark Rd., MS 5-11

${ }^{\S}$ Research Investigator, Department of Aerospace Engineering, AIAA Senior Member

"Professor, Department of Aerospace Engineering, AIAA Associate Fellow 


\section{Introduction}

We are interested in optimizing the performance of a supersonic aircraft when considering its mission profile, thermal systems, and engine design. To successfully design all of these disciplines simultaneously, we need to construct a complex interconnected model that can be used in a gradient-based optimization problem. We have previously performed aero-thermal-mission optimization of a supersonic aircraft [1]. To extend that work to include the design of the engine, we first examine a multipoint engine design problem in this current paper.

Designing a supersonic aircraft to perform well at all of the flight conditions within a nominal mission is challenging [2]. A supersonic aircraft might need to take off in a very short distance, climb to a high altitude quickly, or loiter for a long time [3,4]. We can increase aircraft performance through many methods, such as improving the aerodynamics, using more advanced materials, or using more efficient engines. One way to greatly increase effectiveness is to improve engine performance, which lowers fuel consumption and decreases the aircraft weight for a given mission. To help make the engines more efficient across many flight conditions, researchers previously developed and evaluated variable cycle engines (VCEs) to help tailor performance across the flight envelope [5, 6, 7, 8, 9].

To computationally model engines, methods range from using empirically fit rubber engine models used in preliminary aircraft design to performing unsteady computational fluid dynamics (CFD) on engine components. A popular middle-ground is one-dimensional mean line flow methods, which is what is used in industry-accepted Numerical Propulsion System Simulation (NPSS) and NASA's pyCycle. We want to analyze the performance of the engine across the entire mission but this is prohibitively computationally expensive. Instead of explicitly querying the engine model at all of the relevant points, we can instead look at only the limiting design cases.

Performance at each of these flight conditions is a function of both the physical and operational variables for the engine. Physical variables are those that are considered when doing conventional engine design and include design variables such as fan pressure ratio (FPR), overall pressure ratio (OPR), and extraction ratio (ER). Operational variables are any non-fixed parameters than can change for each flight condition, including any VCE parameters, fuel-to-air ratios, or cooling parameters. The inclusion of these operational variables allows the engine performance to be tailored for each flight condition but greatly increases the design complexity of the model. The optimal engine design found when considering only physical variables is different than the optimal design found when considering both physical and operational variables.

We can use numerical optimization to find the best performance when varying the physical and operational variables. Engine optimization has been used in a variety of contexts, including propulsion-airframe integration [10, 11], multidisciplinary design optimization (MDO) of an aircraft including its heat loads [12], or for boundary layer ingestion [13]. The large design space introduced by the VCE technology necessitates gradient-based optimization. By using the derivative information with a gradient-based method, we can obtain the optimal result in much less computational time $[14,15]$. Some studies have looked at gradient-based optimization of engines [13, 16], but they did not consider VCEs or supersonic engines.

Here we find the optimal design when considering the physical and operational variables for a multipoint problem using gradient-based optimization. We do this by constructing a two-spool mixed-flow turbofan model and exploring the design space through parameter sweeps. We then perform multiple optimizations considering one on-design condition and four off-design conditions simultaneously. We use an open-source computational framework that enables us to use this model in an MDO context for future studies.

\section{Modeling Tools}

\section{A. OpenMDAO}

We use NASA's OpenMDAO [17] as the computational framework because of its modularity and derivative-focused data-passing capabilities. Models constructed in OpenMDAO consist of groups and components, where each component contains a small portion of the total analysis. OpenMDAO uses the modular and unified derivative theory [18] to compute the total derivatives of the system by combining the partial derivatives of individual components. Although OpenMDAO can use a few different optimization methods, we use the gradient-based method SNOPT [19] for all results in this work.

Many features of OpenMDAO helped enable the research presented in this paper. Problem setup was greatly simplified by the ability to stamp out multiple copies of the same analysis group and then connect relevant param- 
eters between the on-design and off-design groups. OpenMDAO handles the data- and derivative-passing automatically, which makes these connections simple to implement. Tools for model layout visualization that come packaged with OpenMDAO decreased the time to set up the problem. Solver convergence information and model debugging tools helped us determine what to fix when the model did not converge successfully. These tools and features make OpenMDAO a useful tool for setting up, analyzing, and optimizing multidisciplinary systems.

\section{B. pyCycle}

The state-of-the-art propulsion modeling tool, NPSS, solves a large nonlinear system of equations that represent the physics from 1-D propulsion models. Despite not providing derivatives, NPSS has been used for optimization by finite differencing across the model. However, many researchers have noted problems with using finite differences across NPSS in an optimization context.

This drove the development of pyCycle [16], a NASA tool that models the same physics as NPSS, but provides analytic derivatives throughout the entire process. Additionally, pyCycle is written in OpenMDAO, which helps support modular model construction and integration into larger MDO problems. pyCycle uses chemical equilibrium analysis [20] for its underlying thermodynamic library and uses a system of nested Newton solvers to converge complex engine cycles efficiently. pyCycle has been used for propulsion-mission optimization [21] and boundary layer ingestion studies [22, 23, 13].

\section{Engine Model}

We have constructed a two-spool mixed-flow turbofan engine model within pyCycle which consists of 21 subparts, or 'elements' from NPSS terminology. This model is based on the GE F110 engine, but only loosely because we vary the design parameters throughout this study. We added VCE parameters, where we can control the inlet guide vanes (IGV) and the variable area bypass injector (VABI) to affect the engine performance.

\section{A. Mixed flow turbofan}

Turbofan engines are used in multiple different classes of aircraft, including commercial airliners of all sizes and many supersonic jets. High-bypass turbofan engines are used in commercial aircraft due to their low thrust-specific fuel consumption (TSFC), while low-bypass turbofan engines are used in supersonic aircraft due to their ability to produce a large amount of specific thrust. In this work, we have constructed a two-spool mixed-flow turbofan engine capable of supersonic flight. It is loosely based on the GE F110 engine, though the design parameters and performance scalars on the performance maps do not match the GE F110. Instead, we use the same element configuration as a GE F110 model to create a generic supersonic engine where we vary the static design. Figure 1 shows how these elements are laid out and connected.

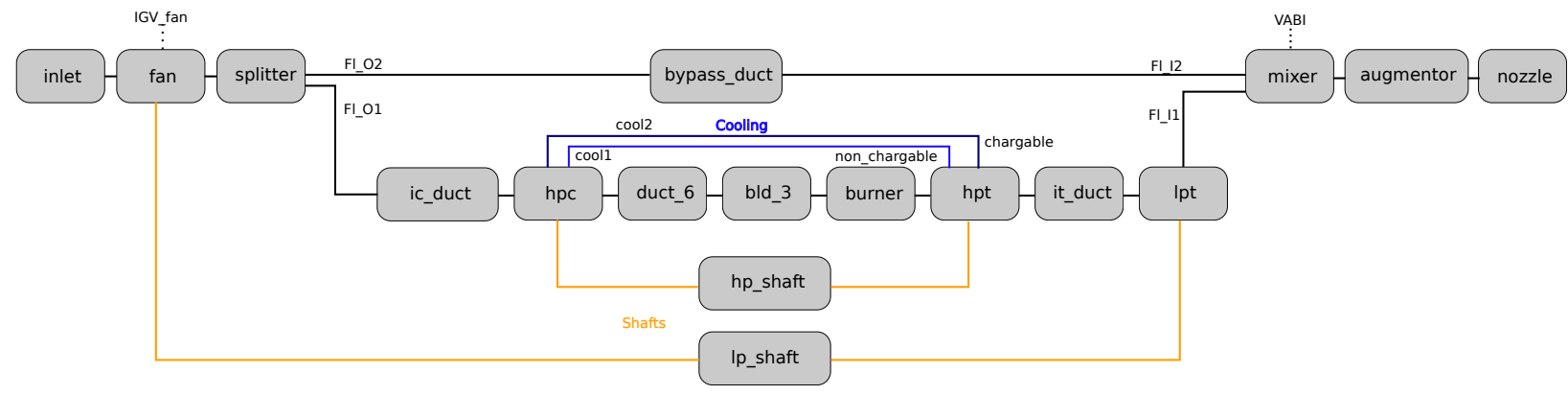

Figure 1. The elements in the mixed-flow turbofan engine model are shown with the VCE parameters labeled as IGV fan and VABI.

Throughout this work we are considering the uninstalled performance of the engine. This is a first step before considering a more complete model where we include installation effects which will give more accurate performance 
data for the engine. Additionally, the model presented here does not include the use of an afterburner. We are designing the engine to meet all of the thrust requirements without an afterburner.

We use performance maps for the compressors and turbines to obtain relevant performance parameters for these elements. Specifically, these maps relate the pressure ratio, corrected mass flow rate, corrected engine speed, and component efficiency for the element $[24,25]$. These maps are then scaled to meet requirements set on the design condition, and these scalars are passed to the off-design conditions where the scaled maps are used to obtain relevant performance parameters.

As alluded to in the previous paragraph, the setup of the problem and how we solve for the states in the engine differ in design and off-design modes. An engine is conventionally designed at a single operating point, sometimes the sea-level static (SLS) condition, but we are interested in its performance at other flight conditions as well. We can vary the fuel flow through the engine to control the thrust, and then solve for the shaft speeds to ensure the engine performance can be calculated correctly. The mass flow rates, net power output, pressure rises, and rotational speeds of the shafts and flows for each compressor and turbine pair must match [25]. This leads to a complex solver hierarchy within this model due to the way we ensure the engine is correctly balanced. We drive the difference between the shaft powers and speeds to 0 using nested Newton systems within the model.

Because of the complex nested solver structure within this model, as well as the actual physics we are modeling, the initial guesses inputted into the model are extremely important. Without good initial guesses, the Newton solvers cannot reach the basin of convergence, and then the model does not converge successfully. To alleviate this problem, we worked to make the engine model robust across a range of flight conditions. This included setting up the internal solvers to have reasonable bounds on the variables, using preconditioners on the linear systems, and improving the Newton linesearch algorithm within OpenMDAO. Additionally, we converged the model at many flight conditions and saved the internal solver values at the converged states. We then set up a surrogate model using this information as training data. We can then query this surrogate model when we run the engine model at a given flight condition and get a reasonable initial guess to use in the engine model, even as we change the static design of the engine. The Mach-altitude points used to set up this surrogate are shown in Fig. 2 to illustrate the flight envelope in which we converged the baseline engine model.

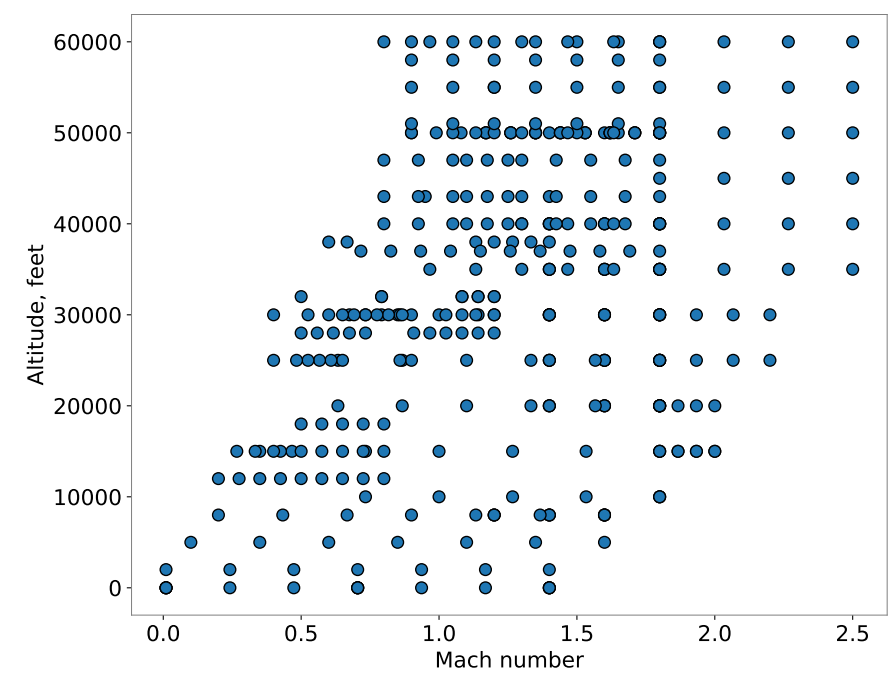

Figure 2. We ran a nominal model at a set design thrust across the Mach-altitude space to determine good initial guesses for the model. 


\section{B. Design variables}

We include five physical variables to control the design of the engine and we will now detail those that we use in this work. OPR is the total pressure ratio of both compressor stages, which is the ratio of the stagnation pressure at the front to the fan to the stagnation pressure at the back of the high-pressure compressor (HPC). FPR is the pressure ratio just across the fan. OPR is equivalent to the product of HPC pressure ratio and FPR. ER is the ratio of the pressure levels in the bypass to the core exhaust ducts. Another design parameter is the desired thrust of the engine

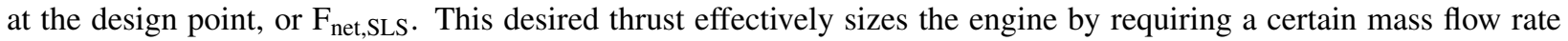
and fuel-to-air ratio (FAR), which in turn scales the performance maps, and changes performance based on the design thrust. FAR influences how much fuel is added in the burner, which is the main driver for thrust production from the engine. Although FAR is a design variable in this work, it is effectively constrained by $T_{4}$, the temperature of the flow leaving the burner. Increasing FAR means more fuel is burned, which means the flow leaving the burner is at a higher temperature. $T_{4}$ is limited by the available material technology level for the high-pressure turbine blades.

Based on these physical design variables, we can obtain an engine design that can be used at off-design points to obtain its performance. These variables are also called static design variables, because they cannot vary at different flight conditions and are fixed for a given engine design. For non-VCE engines, only these physical variables can be varied to change the geometry of the engine.

\section{Operational variables}

We include two operational variables that vary the geometry of the engine model so it can be controlled at individual flight conditions. For the fan, we can control the IGV, which can be thought of as varying the stator vane angle within the compressor. Varying these vane angles mostly affects the performance of the corresponding compressor element, though only for some specific flow parameters. The other operational variable in this model is the VABI, which effectively controls the bypass ratio of the engine by changing the flow areas at the mixer element. This has a large effect on the performance of the entire engine. The combination of these two variables allows the BPR, stall margins, and mass flow rates to be tailored for each flight condition queried using the engine model.

We now discuss how these VCE parameters are implemented in the engine model. The IGV control is achieved by querying the compressor maps accounting for the vane angles. Specifically, we supply the corrected speed, Rline value, and IGV value to the compressor map to obtain the corrected flow, efficiency, and pressure ratio. In essence, the ability to control the IGV comes from having the correct maps that have this information tabulated. On the other hand, the VABI control does not deal with any elements maps. Instead, we directly vary the areas in the mixer element, using the design case areas as the baseline. As the VABI control is increased, the area for the core flow in the mixer is increased and the area for the bypass flow is decreased. As the VABI control is decreased, the opposite is true. The total area in the mixer remains fixed and does not change based on the VABI setting.

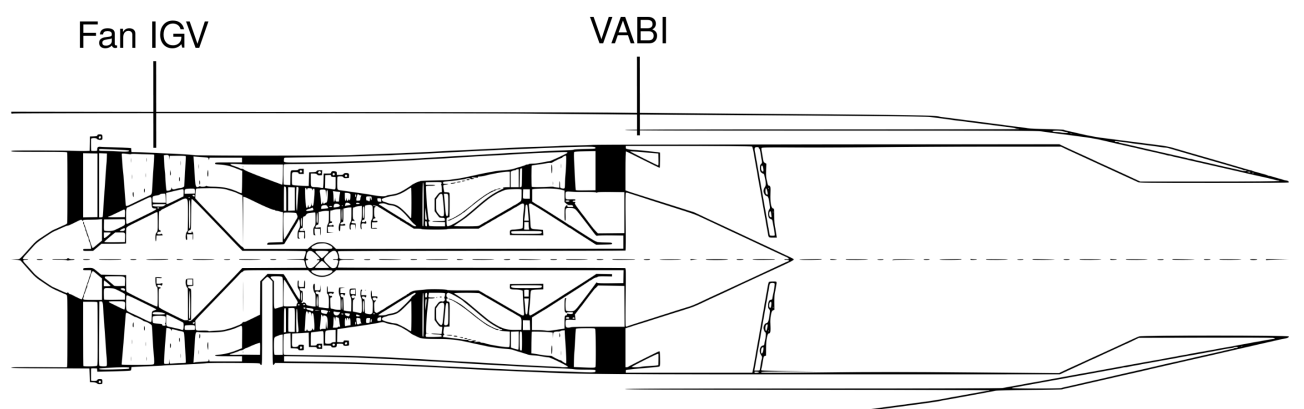

Figure 3. A cutout view of a representative engine with the IGV and VABI controls highlighted. Adapated from Heath et al [10].

In addition to these two VCE variables, we can also vary FAR at each of the off-design points to hit a thrust target. Although FAR is not considered a VCE parameter, it is an operational variable that can be changed to improve the performance at each flight condition. 


\section{Parameter sweeps}

With this model constructed, we performed sweeps on some of the operational variables to better understand the design space. This lets us see the effects of the changing the IGV and VABI controls to see how the performance can be tuned at different flight conditions. The effects of these operational variables depend on the physical design parameters and where the engine is operating in Mach-altitude space. These are complex relationships and difficult to generalize, but here we present two different parameter sweeps to examine a subset of the trends we expect to see. Both sweeps are at the same physical design and Mach-altitude flight condition. Specifically, here the engine is sized at 17,000 lbf for the SLS design case and we are performing the sweeps at Mach 1.6 and an altitude of 50,000 ft. Each of the y-axes in the plots shown in this subsection are normalized by the baseline value. For IGV, this is the value when the control is set to 0.0, while for the VABI this corresponds to when the control is set to 1.0. This is done to show the relative variation instead of dimensional variation in each of these outputs to make these results easier to generalize for other flight conditions.

Figure 4 show the effect of sweeping through the fan IGV parameter from its lower to upper bounds. We see negligible changes in mass flow rate, but larger changes in corrected speed. This means that the IGV parameter varies where the compressor is operating on its performance map. Due to the IGV affecting where on the map the element is operating, we see a relatively change in the stall margin. If the physical engine design puts one of the operating points on the corrected speed or stall margin constraint, we could change the corresponding IGV parameter to move the operating point off that constraint and into the feasible region. To a first order, varying the IGV allows control of the stall margin and corrected speed. The small kinks in the mass flow rate curve come from the linear interpolation used when reading in the performance maps for the elements and has a negligible effect on the engine.

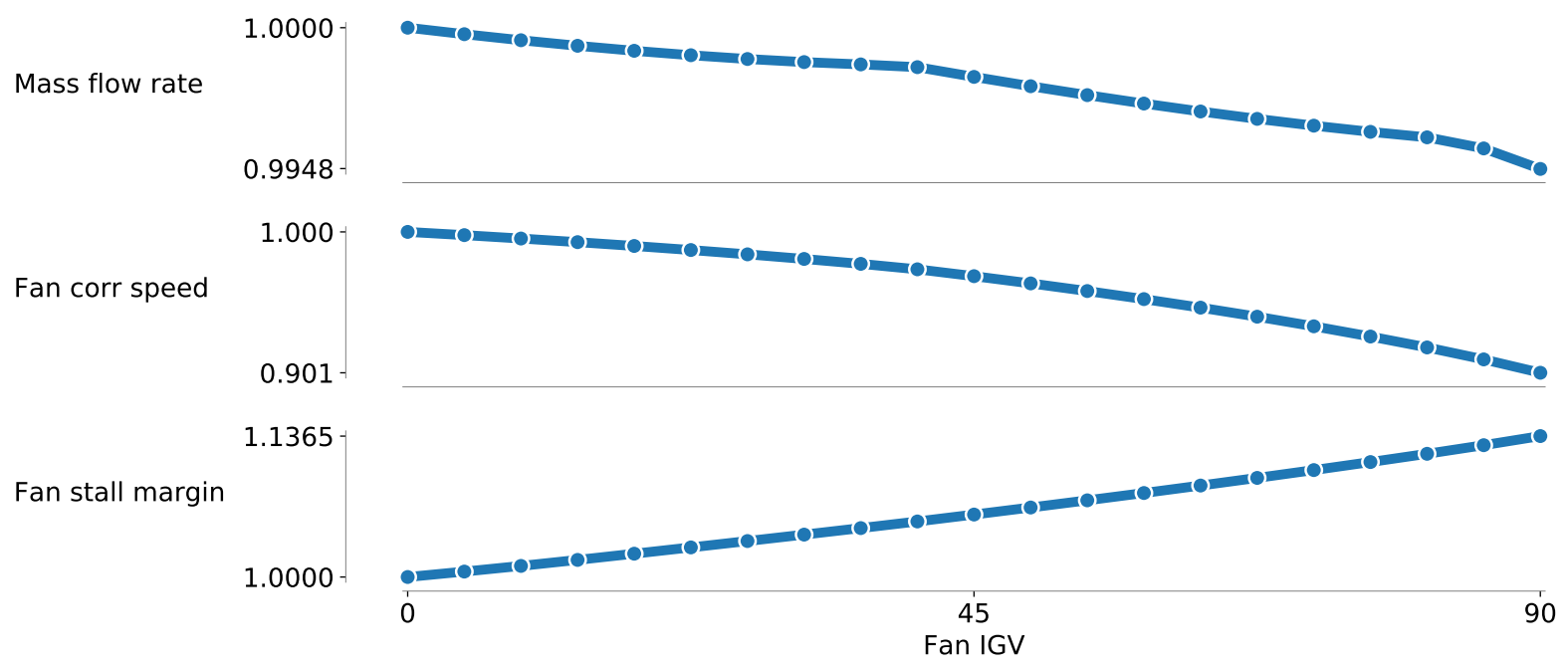

Figure 4. The fan IGV controls the stall margin, but we also see a change in corrected speed.

Unlike the IGV, the VABI has a larger affect on the overall performance of the engine. As we increase VABI, we are increasing the area in the mixer corresponding to the core flow while we lessen the area for the bypass flow. Thus, the total area remains the same as VABI changes. Looking at Fig. 5, as VABI increases, we see BPR, net force, and mass flow rate increase noticeably, while FAR decreases slightly.

The fact that BPR increases as we increase VABI is counterintuitive, and we now investigate the physical reasons behind this trend. As we increase the area in the core, the back-pressure decreases, which decreases the pressure drop across the turbine. This lower pressure ratio means that the high-pressure turbine puts less power on the shaft, which effectively slows down the shaft. However, this also means there was more energy left in the flow when it encounters the low-pressure turbine, which causes that turbine shaft to increase in speed, as we see in the bottom portions of Fig. 5 . 
This increase in fan shaft speed increases the amount of flow that goes through the bypass duct, which increases BPR.

Whereas the IGV mostly control some aspects of the flow and engine performance near their respective elements, the VABI can be seen as an overall engine performance parameter. We expect the optimizer to take advantage of these coupled effects between operational parameters where a human designer would have trouble intuitively understanding the optimal settings for each of the flight conditions due to the complexity of interactions.

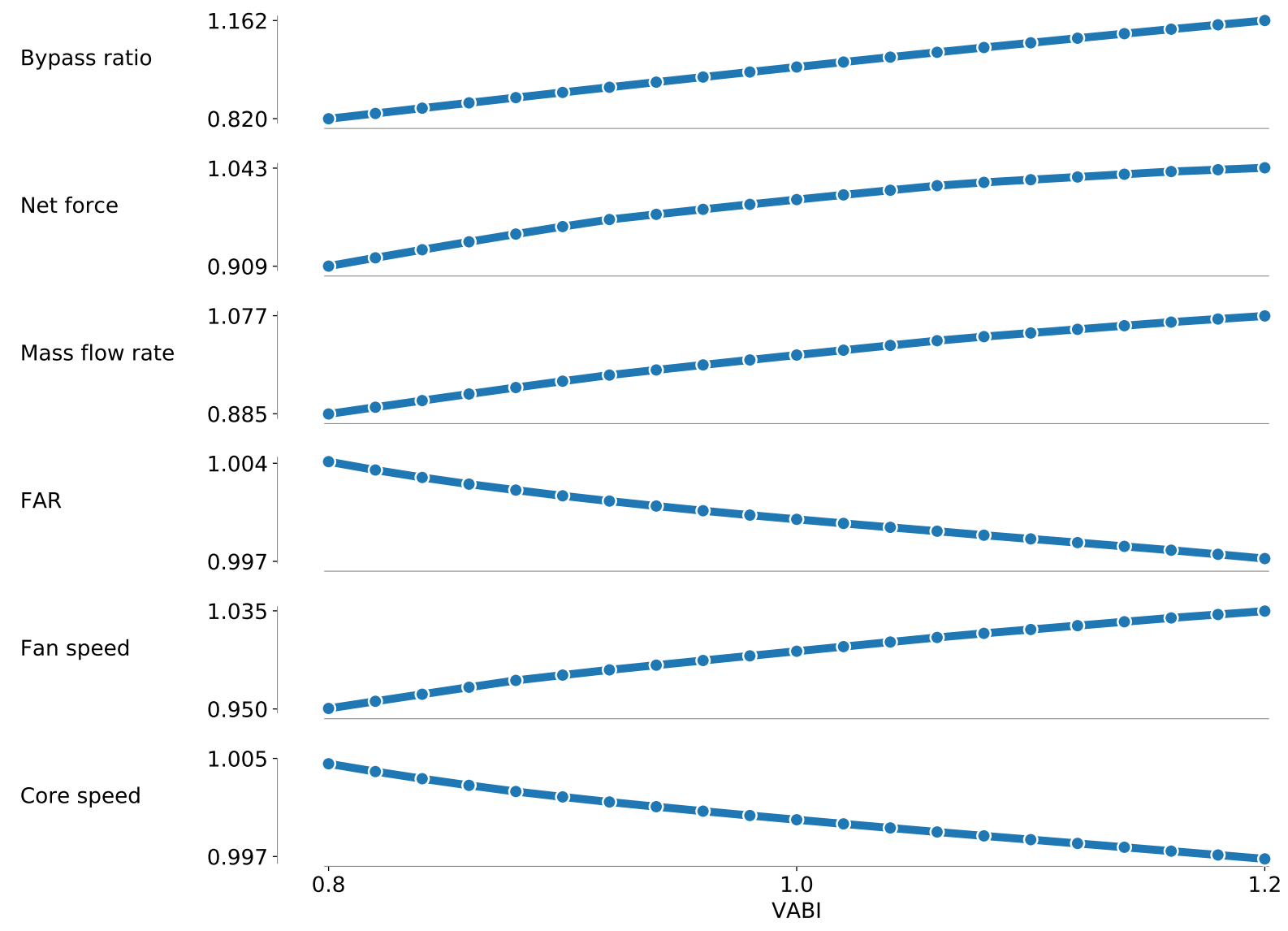

Figure 5. We see a greater effect on performance from the VABI compared to the IGV.

After examining how the VABI affects performance at a single flight condition, we also examined the performance across three Mach-altitude combinations. Figure 6 shows the fan map with points from three different VABI sweeps overlaid on the map. In general, as we increase Mach number and altitude, the fan moves to a lower corrected speed and lower pressure ratio. As we increase the VABI, we see that the points may become choked depending on the flight condition. Additionally, the efficiency of the fan decreases as we increase VABI here. This decrease in efficiency may result in better overall engine performance depending on the flight conditions and constraints imposed. We expect the optimizer to take advantage of this freedom by tailoring the VABI parameter at each off-design condition. 


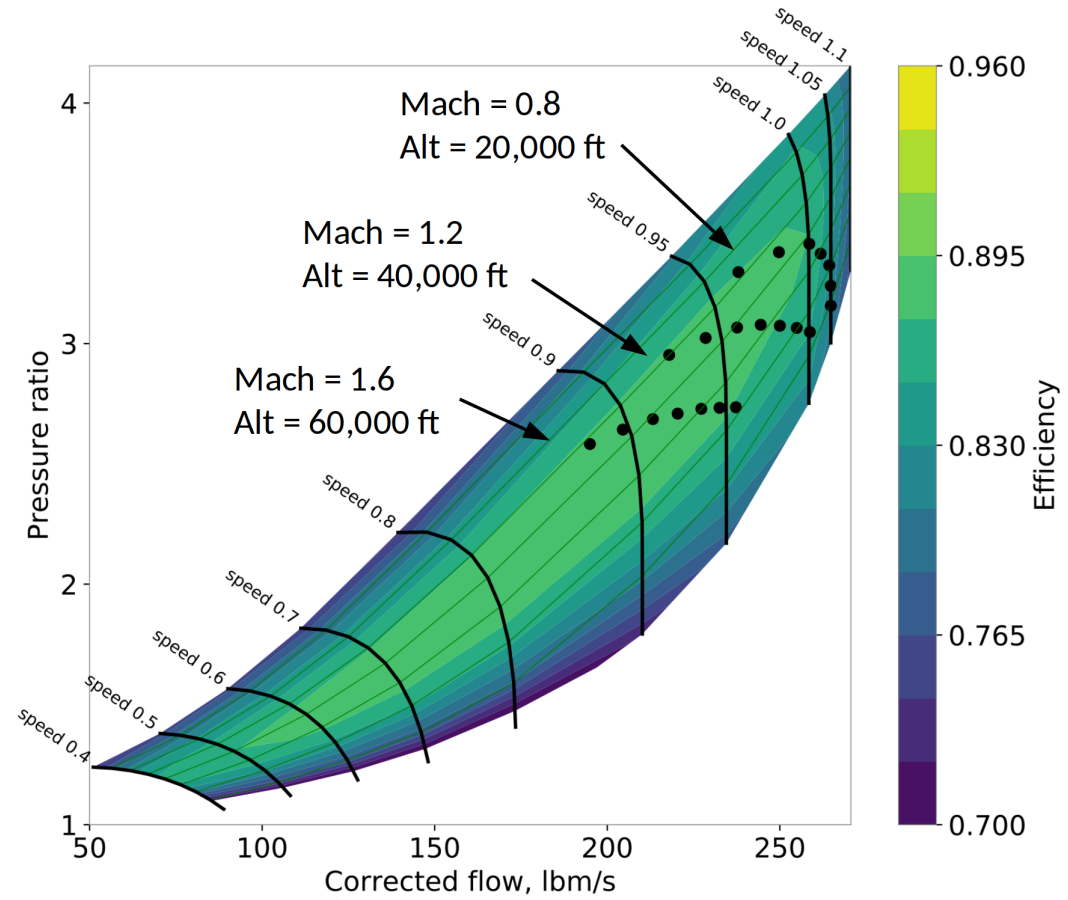

Figure 6. We see the fan efficiency vary greatly based on the flight condition and VABI setting. The VABI setting sweeps from 0.8 for the leftmost points to 1.2 for the rightmost.

\section{Optimization Problem Formulations and Results}

Now that we have constructed a model, briefly explored its design space, and have the ability to compute gradients through the entire system, we can formulate the optimization problems we want to solve. We first determine the flight conditions to use in this multipoint engine design problem, then we detail how we chose the design variables, constraints, and objectives for the optimization problems before presenting results.

\section{A. Flight condition selection}

Given that we do not wish to analyze every point along the mission profile, we need to find the critical points in the flight envelope where the engine is constrained in some way. The physical reason for these constraints might primarily come from aircraft, engine, or mission requirements. For example, if our aircraft is at its maximum takeoff weight (MTOW) and is on a short runway, we would need to produce a large amount of thrust to accelerate the heavy aircraft quickly enough to safely takeoff. Another source for performance limits comes from the maximum temperature allowed at the exit of the burner, or $T_{4}$ as it is commonly known. This is generally limited by the material of the high-pressure turbine blades, which would begin to degrade or melt if exposed to too great of a temperature. Another possible limiting case would be if the desired mission requires a supersonic cruise at high altitude. Nominally, it is challenging to get enough air into the aircraft at high altitudes to produce enough thrust to sustain supersonic flight. On most modern fighter jets, supercruise is possible without the use of afterburners, though afterburners are still used when maximum thrust output is needed.

Although there are many possible representative points, we select rolling takeoff (RTO), top of climb (TOC), subsonic (SC), and after ordinance drop (AOD) as the four off-design conditions where we are trying to optimize engine performance. These points are shown on a supersonic ordinance drop mission in Fig. 7. RTO requires a large amount of thrust at a low altitude and Mach number when the aircraft is fully loaded. TOC needs to be able to produce adequate thrust for a close-to-MTOW aircraft at a moderately high altitude and supersonic Mach number. SC requires 
a small amount of thrust at a transonic Mach number, but we want the TSFC at this point to be low to increase the range of the aircraft. AOD needs a large amount of thrust to produce enough acceleration to quickly disengage after an ordinance drop at low altitude. The aircraft would perform optimally at each one of these flight conditions with a certain engine design, but we want to find the best single design that satisfies all of these constraints while minimizing a certain metric, such as a summation of fuel burn.

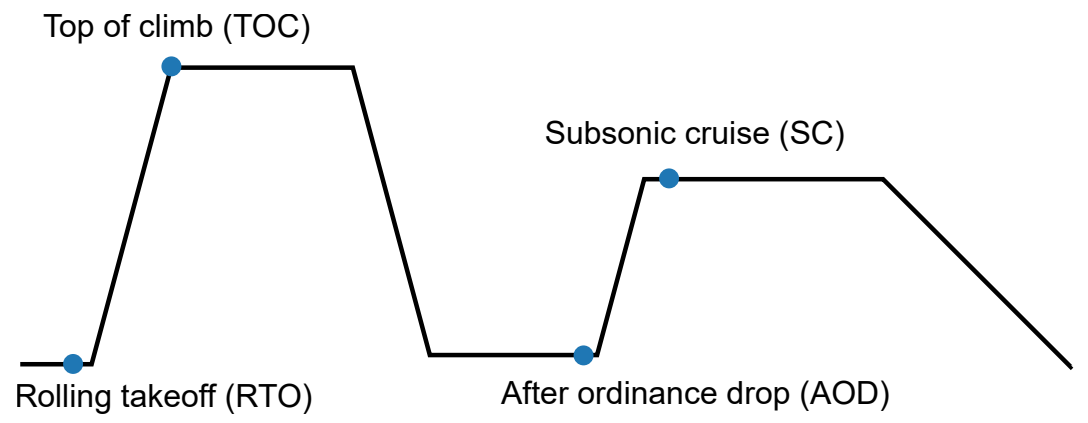

Figure 7. The altitude profile from a nominal mission shows the four flight conditions of interest.

\section{B. Target Thrust Calculation}

Given a certain aircraft weight and aerodynamic performance, we can compute the thrust needed at each one of these flight conditions. We used aerodynamic performance data from a previous study by the authors [1] which was obtained by performing Reynolds-averaged Navier Stokes CFD on the Efficient Supersonic Air Vehicle aircraft. In that previous work, we ran the model at 273 different flight conditions in the Mach-altitude- $\alpha$ design space to construct a 3D surrogate model. Here, we query that model to obtain the lift and drag of the aircraft at our flight conditions of interest.

We use the unsteady aircraft equations of motion to determine the thrust needed at each condition. To do this, we look at the four main force sources on the aircraft — thrust, weight, lift, and drag — and ensure that the vector sum of these forces results in the desired net force on the aircraft to achieve the prescribed motion. In the SC case, we prescribe a net force of 0 on the aircraft, while in the RTO and AOD cases, we prescribe a minimum acceleration. For TOC, we enforce a minimum climb rate related to performance requirements at the aircraft's service ceiling. We then solve these equations by varying thrust and angle of attack to satisfy these constraints and obtain the minimum thrust values needed for each condition. Explicitly, we use these equations of motion,

$$
\begin{aligned}
& \dot{v}=T \cos (\alpha)-D m-g \sin (\gamma) \\
& \dot{\gamma}=\frac{T \sin (\alpha)+L}{m v-(g / v) \cos (\gamma)} \\
& \dot{h}=v \sin (\gamma) \\
& \dot{r}=v \cos (\gamma),
\end{aligned}
$$

where $v$ is the aircraft velocity, $T$ is the thrust, $\alpha$ is the angle of attack, $D$ is the drag, $L$ is the lift, $m$ is the aircraft mass, $g$ is the acceleration due to gravity, $r$ is the aircraft range, and $\gamma$ is the flight path angle. 
Table 1. Tabulated data for each flight condition, with the thrusts obtained from solving the nonlinear set of equations.

\begin{tabular}{lrrrrrr}
\hline Flight condition name & Mach & Altitude, ft & Weight, lb & Acceleration, ft/s & Climb rate, fpm & Thrust, lbf \\
\hline Rolling takeoff (RTO) & 0.2 & 0.1 & 68,000 & 19.9 & 0. & 28,000 \\
After ordinance drop (AOD) & 0.6 & 1000 & 50,000 & 24.6 & 0. & 22,450 \\
Subsonic cruise (SC) & 0.8 & 35,000 & 66,000 & 0. & 0. & 2060 \\
Top of climb (TOC) & 1.4 & 50,000 & 50,000 & 0. & 800. & 7600 \\
\hline
\end{tabular}

The resulting minimum thrust values are shown in Tab. 1. We use two engines to power the aircraft and the thrust values shown are for a single engine. We see that the RTO and AOD cases require the most thrust because we are prescribing minimum accelerations. Additionally, the TOC case requires a large amount of thrust for its altitude. We do not know a priori which case will be the most limiting for the engine design.

\section{Design variables, constraints, and objective}

We parameterized this model to have five physical design variables that control the size and performance of the engine. These design variables are applied at the on-design condition, SLS, and the resulting design is then passed to each of the off-design cases. We allow the optimizer to vary the net thrust, the mixer ER, FPR, OPR, and FAR in the core. We selected these design variables to produce a well-posed optimization problem that gives enough freedom to the optimizer to control the overall engine performance. In addition to these physical variables, we also give the optimizer freedom to vary the operational variables at each flight condition. This includes the fan IGV, VABI, and FAR at each point.

We add constraints to the optimization problem to produce a realistic design. We constrain the area of the inlet to limit the overall size of the engine. Because we are not designing the entire aircraft, this area constraint is in place to limit the size of the engine so it could fit within an existing airframe. A more complete optimization problem would at least consider the supersonic inlet, if not size the aircraft at the same time, but that is outside the scope of the current work. We also constrain the flow exit temperature of the burner at all flight conditions by an upper bound due to the material of the high-pressure turbine blades. At each flight condition we constrain the corrected speed of the fan to not exceed the maximum speed allowed by the engine assembly. We also add minimum thrust targets for each of the off-design points.

We could select an objective function from a few quantities of interest, including fuel burn, MTOW, maximum speed, maximum altitude, etc. Given that we are not designing the entire aircraft in this work, we chose to minimize an equally-weighted sum of TSFC values at the four off-design points to obtain a representative measure of the performance of the engine. This is not necessarily a realistic design objective, but we use it here to illustrate how the model and optimization method consider performance across multiple flight conditions simultaneously. A more advanced optimization problem might consider varying the aerodynamic properties, the internal structure, or the mission profile of the aircraft simultaneously as we design the engine.

Table 2 shows the complete optimization problem formulation for two cases. In the first case, we give the optimizer freedom to vary all operational variables, whereas in the second case, we remove the VCE parameters. This allows us to see the effect that the VCE parameters have on the optimal performance of the engine. Although FAR is considered an operational variable, we include it in both problem formulations because non-VCE cycles can vary FAR at each off-design point.

The first five variables in Tab. 2 correspond to the physical design variables for the engine, specifically at the SLS case we selected as our design point. The rest of the variables are the operational variables for each off-design point, including the two VCE parameters as well as FAR. We then have the inlet area, burner exit temperature, and corrected speed constraints, which help keep the engine within a realistic physical limits. the minimum thrust targets for the off-design points fill out the bottom of the table. This gives us a total of 18 design variables and 15 constraints for the full problem. 
Table 2. This shows the multipoint optimization problem formulations for both the full multipoint problem considering all operational variables as well as the multipoint problem where we do not vary the VCE parameters.

\begin{tabular}{llrrrrl}
\hline Category & Name & Quantity - VCE & Quantity - No VCE & Lower & Upper & Units \\
\hline Objective & $\sum$ TSFC & 1 & 1 & - & - & $\frac{\mathrm{lbm}}{\mathrm{hr} \text { lbf }}$ \\
\hline Variables & FPR & 1 & 1 & 2.5 & 4.0 & - \\
& ER mixer & 1 & 1 & 0.9 & 1.1 & - \\
& OPR & 1 & 1 & 20. & 35. & - \\
& $F_{\text {net,SLS }}$ & 1 & 1 & 15,000 & 35,000 & lbf \\
& FAR & 1 & 1 & 0.015 & 0.028 & - \\
& FAR & 1 & 4 & 0.0135 & 0.028 & - \\
& IGV & 4 & 0 & 0. & 90. & - \\
& VABI & 4 & 0 & 0.95 & 1.05 & - \\
& Total & 4 & $\mathbf{1 0}$ & & & \\
\hline Constraints & Inlet area & $\mathbf{1 8}$ & 1 & 1000. & 2000. & inches \\
& $T_{4}$ & 1 & 5 & 2000. & 3200. & ${ }^{\circ} \mathrm{R}$ \\
& Fan corrected speed & 5 & 5 & 0.4 & 1.05 & - \\
& $F_{\text {net,RTO }}$ & 5 & 1 & 28,000 & - & lbf \\
& $F_{\text {net,TOC }}$ & 1 & 1 & 7600 & - & $1 \mathrm{bf}$ \\
& $F_{\text {net,SC }}$ & 1 & 1 & 2060 & - & $1 \mathrm{bf}$ \\
& $F_{\text {net,AOD }}$ & 1 & 1 & 22,450 & - & lbf \\
& Total & 1 & $\mathbf{1 5}$ & & & \\
\hline
\end{tabular}

\section{Optimization results}

We performed six different individual optimizations using the VCE cycle, each one with a different maximum allowed inlet area, and repeated these six optimizations with the non-VCE cycle. By varying the area constraint and optimizing the physical and operational variables, we can directly see the effect that this constraint has on the optimal design and performance of the engine. Recall that all analysis done here is considering the uninstalled performance of the engine and we do include an afterburner. Because we do not have a measure of the effects of the inlet geometry, we use the inlet area constraint as a stand-in.

Results from these optimizations are shown tabularly in Tab. 3 and graphically in Fig. 8. As we make the inlet area constraint more restrictive, we see that the optimizer decreases the design case net thrust. However, since we are still enforcing the minimum thrust constraints on the off-design points, the optimizer increases FPR and decreases BPR to produce more thrust from the core to make up the decreased ability to produce thrust using the bypass stream. As one would expect, as we limit the design space through more restrictive inlet area constraints, the TSFC values increase. In each of the optimization cases, $T_{4}$ is at its upper limit only for the TOC flight condition, whereas the other flight conditions are at partial throttle or are below the $T_{4}$ limit.

At each optimal point, the VCE-capable cycle outperforms the non-VCE cycle. This is as expected, since we are giving the optimizer more freedom to vary the engine design at each flight condition when using VCE parameters. The non-VCE optimization corresponding to an inlet area of $1500 \mathrm{in}^{2}$ did not converge because the optimizer could not find a feasible point, so we do not show results for that case. 
Table 3. Optimized design variables and performance obtained from each of the six optimization problems for the VCE cycle.

\begin{tabular}{rrrrrrrrr}
\hline Inlet area, in & $\mathrm{F}_{\text {net,SLS }}, \mathrm{lbf}$ & $\mathrm{ER}_{\text {mixer }}$ & $\mathrm{OPR}$ & $\mathrm{FPR}$ & $\mathrm{TSFC}_{\mathrm{SC}}, \frac{\mathrm{lbm}}{\mathrm{hl} \mathrm{lbf}}$ & $\mathrm{TSFC}_{\mathrm{TOC}} \frac{\mathrm{lbm}}{\mathrm{hr} \mathrm{lbf}}$ & $T_{4, R T O},{ }^{\circ} \mathrm{R}$ & $T_{4, T O C},{ }^{\circ} \mathrm{R}$ \\
\hline 2000 & 33,435 & 0.942 & 35.0 & 2.855 & 0.703 & 0.882 & 3070.7 & 3200.0 \\
1900 & 32,873 & 0.945 & 35.0 & 2.969 & 0.712 & 0.895 & 3002.5 & 3200.0 \\
1800 & 32,301 & 0.947 & 35.0 & 3.103 & 0.722 & 0.907 & 3013.5 & 3200.0 \\
1700 & 31,685 & 0.954 & 35.0 & 3.239 & 0.733 & 0.921 & 3024.4 & 3200.0 \\
1600 & 30,818 & 0.956 & 35.0 & 3.369 & 0.745 & 0.939 & 3029.9 & 3200.0 \\
1500 & 30,650 & 0.963 & 35.0 & 3.630 & 0.761 & 0.955 & 3052.5 & 3200.0
\end{tabular}

TSFC $_{\text {supersonic, }} \mathrm{lbm} /(\mathrm{h} * \mathrm{lbf})$

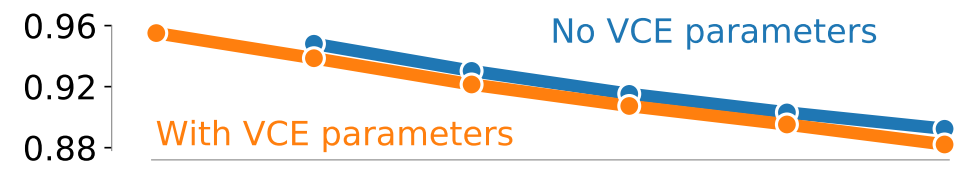

$\mathrm{TSFC}_{\text {subsonic, }} \mathrm{Ibm} /(\mathrm{h} * \mathrm{lbf})$

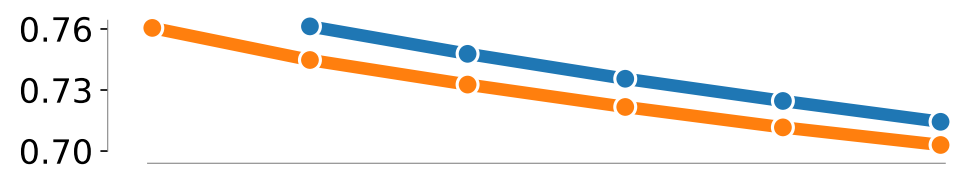

Mass flow rate, $\mathrm{lbm} / \mathrm{s}$

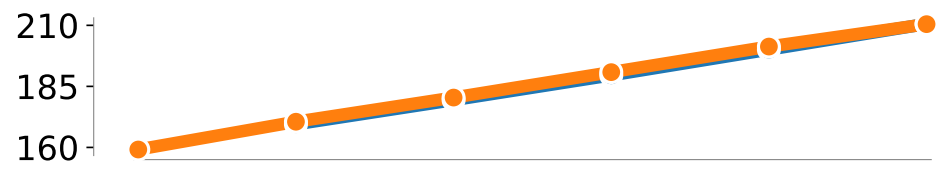

Bypass ratio

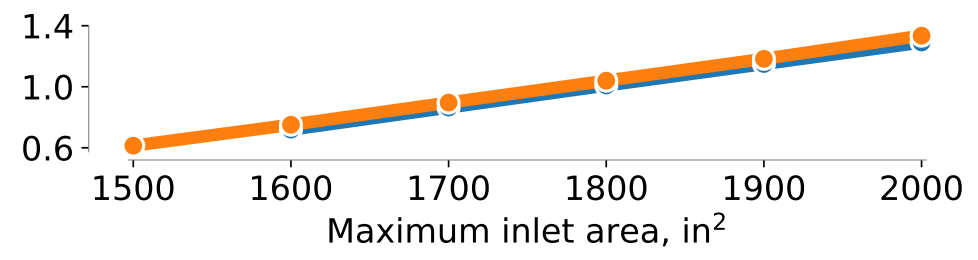

Figure 8. As we decrease the allowable inlet area, TSFC increases and mass flow rate and bypass ratio decrease.

The operational parameters are shown in Tab. 4. Although they varied during the optimization process, the IGV and VABI settings are all on a bound at the optimal design. This is partially due to our objective function, which seeks to minimize TSFC across all points, which encourages the optimizer to maximize the VABI setting because we are not considering installed effects. Because we did not include compressor stall margin constraints, the IGV are on their lower bounds at the optimal points, which minimizes TSFC. We would expect these optimal operational parameters to move off the bounds if we had a more realistic problem formulation with additional constraints. 
Table 4. Operational variables at the optimal points for each of the six optimization problems, looking at only the minimum and maximum values across the four flight conditions.

\begin{tabular}{rrrrrrr}
\hline & \multicolumn{2}{c}{ IGV $_{\text {Fan }}$} & \multicolumn{2}{c}{ VABI } & \multicolumn{2}{c}{ FAR } \\
\cline { 2 - 7 } Inlet area, in & Min & Max & Min & Max & Min & Max \\
\hline 2000 & 0.000 & 0.031 & 1.049 & 1.050 & 0.0178 & 0.0264 \\
1900 & 0.000 & 0.023 & 1.050 & 1.050 & 0.0173 & 0.0262 \\
1800 & 0.000 & 0.028 & 1.050 & 1.050 & 0.0168 & 0.0262 \\
1700 & 0.000 & 0.083 & 1.050 & 1.050 & 0.0161 & 0.0262 \\
1600 & 0.000 & 0.026 & 1.050 & 1.050 & 0.0159 & 0.0260 \\
1500 & 0.000 & 0.072 & 1.050 & 1.050 & 0.0158 & 0.0262
\end{tabular}

We also performed four single-point optimizations to compare against the multipoint optimized result. All optimizations for this comparison used an inlet area constraint of $1800 \mathrm{in}^{2}$. In each single-point optimization, we ran only the on-design and one off-design flight condition without considering the performance of any other flight condition. We gave the optimizer control over all of the physical and operational variables corresponding to that single flight condition of interest and retained the same constraints on that flight condition as in the multipoint problem. The objective function for each of these single-point optimizations was the TSFC of that individual point. That is, the results from each of these single-point optimizations is the best case scenario in terms of performance at a given flight condition. Figure 9 shows the TSFC values obtained from the VCE-capable multipoint optimization as well as four individual single-point optimization. We see that the single-point TSFC values outperform the multipoint results across all four flight conditions. This allows us to quantify the sacrifice in fuel consumption efficiency necessary to meet performance demands at different flight conditions.

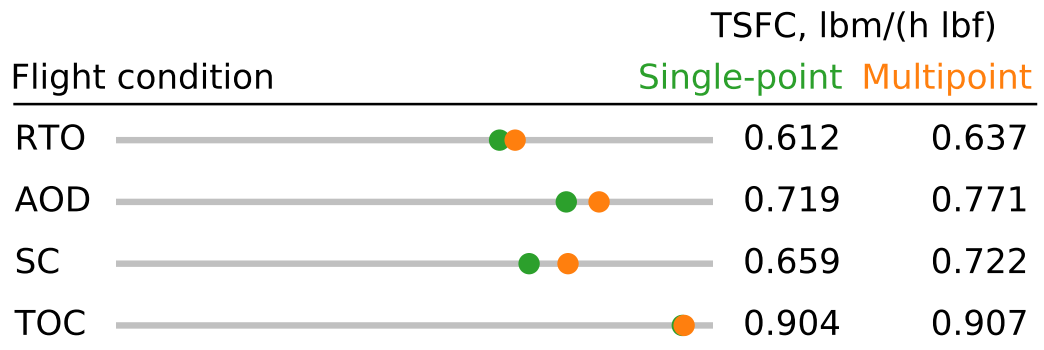

Figure 9. The single-point optimizations result in TSFC values that are 0.3-8.6\% lower than those from the multipoint optimization, showing the decreased fuel efficiency at individual flight conditions when we optimize considering multiple flight conditions.

\section{Conclusion}

We created a supersonic mixed-flow turbofan engine model that provides analytic gradients of its performance with respect to physical and operational variables. Parameter sweeps across the VCE variables showed we can tailor the performance of the engine individually at each flight condition. This model was used in multiple optimization problems where we found the optimal design in a multipoint context using a gradient-based method to minimize equally-weighted summed TSFC. The full optimization problems had 18 design variables representing the physical and operational parameters, as well as 15 constraints to keep the design physically reasonable and meet thrust requirements. We found that as we restrict the inlet area, the TSFC at each flight condition increases, leading to overall increased fuel consumption.

A few improvements to the model and optimization problems could greatly improve the quality of results. Including installed performance would increase the physicality of the problem and allow for more interesting trades in the 
design space, especially considering the VCE parameters. Adding stall margin constraints and considering the afterburner would further improve the realism of this model. Further work could be done to extend this model to possibly include an additional bypass stream, or other variable cycle parameters.

The model presented in this work allows us to explore the complex design space of this supersonic variable cycle engine using gradient-based optimization methods. This engine model can now be integrated into a more complete aircraft design problem to provide realistic propulsion performance as we optimize its design.

\section{Acknowledgements}

The first author is grateful for support from the National Science Foundation Graduate Research Fellowship under Grant No. DGE-1256260. This work is supported in part by the U.S. Air Force Research Laboratory (AFRL) under the Michigan-AFRL Collaborative Center in Aerospace Vehicle Design (CCAVD), with Darcy Allison as the task Technical Monitor. This work is also supported by the NASA Transformational Tools and Technologies Project, under the Aeronautics Research Mission Directorate.

\section{References}

[1] Jasa, J. P., Mader, C. A., and Martins, J. R. R. A., "Trajectory Optimization of a Supersonic Air Vehicle with Thermal Fuel Management System," AIAA/ISSMO Multidisciplinary Analysis and Optimization Conference, Atlanta, GA, June 2018. doi:10.2514/6.2018-3884.

[2] Gertler, J., “F-35 Joint Strike Fighter (JSF) Program,” Library of Congress Washington DC Congressional Research Service, 2012.

[3] Allison, D., Morris, C., Schetz, J., Kapania, R., Sultan, C., Deaton, J., and Grandhi, R., "A multidisciplinary design optimization framework for design studies of an efficient supersonic air vehicle," 12th AIAA Aviation Technology, Integration, and Operations (ATIO) Conference and 14th AIAA/ISSMO Multidisciplinary Analysis and Optimization Conference, 2012, p. 5492. doi:10.2514/6.2012-5492.

[4] Simmons, R. J., Design and control of a variable geometry turbofan with and independently modulated third stream, Ph.D. thesis, The Ohio State University, 2009.

[5] Berton, J. J., Haller, W. J., Senick, P. F., Jones, S. M., and Seidel, J. A., “A comparative propulsion system analysis for the high-speed civil transport," 2005.

[6] Johnson, J., "Variable cycle engines-The next step in propulsion evolution," 12th Propulsion Conference, 1976, p. 758.

[7] Willis, E., "Variable-cycle engines for supersonic cruise aircraft," 1976.

[8] Johnson, J., "Variable cycle engine developments at General Electric 1955-1995," Developments In High-Speed Vehicle Propulsion Systems, 1995, pp. 105-158.

[9] Kurzke, J., "The mission defines the cycle: turbojet, turbofan and variable cycle engines for high speed propulsion," Tech. rep., DACHAU (GERMANY), 2010.

[10] Heath, C., Seidel, J., and Rallabhandi, S. K., "Viscous Aerodynamic Shape Optimization with Installed Propulsion Effects," 35th AIAA Applied Aerodynamics Conference, 2017, p. 3046. doi:10.2514/6.2017-3046.

[11] Rallabhandi, S. K. and Mavris, D. N., "Simultaneous airframe and propulsion cycle optimization for supersonic aircraft design," Journal of Aircraft, Vol. 45, No. 1, 2008, pp. 38-55. doi:10.2514/1.33183.

[12] Allison, D. L., Alyanak, E. J., and Shimmin, K., "Aircraft System Effects Including Propulsion and Air Cycle Machine Coupled Interactions," 57th AIAA/ASCE/AHS/ASC Structures, Structural Dynamics, and Materials Conference, 2016, p. 0671. doi:10.2514/6.2016-0671.

[13] Gray, J. S. and Martins, J. R. R. A., "Coupled Aeropropulsive Design Optimization of a Boundary Layer Ingestion Propulsor," The Aeronautical Journal, 2018. doi:10.1017/aer.2018.120, (In press).

[14] Lyu, Z., Xu, Z., and Martins, J. R. R. A., "Benchmarking Optimization Algorithms for Wing Aerodynamic Design Optimization," Proceedings of the 8th International Conference on Computational Fluid Dynamics, Chengdu, Sichuan, China, July 2014, ICCFD8-2014-0203.

[15] Gronstedt, T. and Wallin, M., "A comparative study of genetic algorithms and gradient methods for RM12 turbofan engine diagnostics and performance estimation," ASME Turbo Expo 2004: Power for Land, Sea, and Air, American Society of Mechanical Engineers, 2004, pp. 615-624. 
[16] Hearn, D. T., Hendricks, E., Chin, J., Gray, J. S., and Moore, D. K. T., "Optimization of Turbine Engine Cycle Analysis with Analytic Derivatives," 17th AIAA/ISSMO Multidisciplinary Analysis and Optimization Conference, part of AIAA Aviation 2016 (Washington, DC), 2016. doi:10.2514/6.2016-4297.

[17] Gray, J. S., Hwang, J. T., Martins, J. R. R. A., Moore, K. T., and Naylor, B. A., “OpenMDAO: An open-source framework for multidisciplinary design, analysis, and optimization,” Structural and Multidisciplinary Optimization, 2019, (Accepted subject to minor revisions).

[18] Hwang, J. T. and Martins, J. R. R. A., "A computational architecture for coupling heterogeneous numerical models and computing coupled derivatives," ACM Transactions on Mathematical Software, Vol. 44, No. 4, June 2018, pp. Article 37. doi:10.1145/3182393.

[19] Gill, P. E., Murray, W., and Saunders, M. A., "SNOPT: An SQP algorithm for large-scale constrained optimization,” SIAM Journal of Optimization, Vol. 12, No. 4, 2002, pp. 979-1006. doi:10.1137/S1052623499350013.

[20] Gray, J. S., Chin, J., Hearn, T., Hendricks, E., Lavelle, T., and Martins, J. R. R. A., "Chemical Equilibrium Analysis with Adjoint Derivatives for Propulsion Cycle Analysis," Journal of Propulsion and Power, Vol. 33, No. 5, September 2017, pp. 1041-1052. doi:10.2514/1.B36215.

[21] Hendricks, E. S., Falck, R. D., and Gray, J. S., "Simultaneous Propulsion System and Trajectory Optimization," 18th AIAA/ISSMO Multidisciplinary Analysis and Optimization Conference, Denver, CO, June 2017.

[22] Gray, J., Mader, C. A., Kenway, G. K. W., and Martins, J. R. R. A., "Modeling Boundary Layer Ingestion Using a Coupled Aeropropulsive Analysis," Journal of Aircraft, Vol. 55, No. 3, May 2018, pp. 1191-1199. doi:10.2514/1.C034601.

[23] Gray, J. S., Kenway, G. K. W., and Martins, J. R. R. A., "Aero-propulsive Design Optimization of a Turboelectric Boundary Layer Ingestion Propulsion System," 2018 AIAA/ISSMO Multidisciplinary Analysis and Optimization Conference, Atlanta, GA, June 2018, AIAA 2018-3976.

[24] Mattingly, J. D., Elements of propulsion: gas turbines and rockets, American Institute of Aeronautics and Astronautics, 2006.

[25] Cumpsty, N. and Heyes, A., Jet propulsion, Cambridge University Press, 2015. 